\title{
Human Evaluation of Machine Translation Through Binary System Comparisons
}

\author{
David Vilar, Gregor Leusch \\ and Hermann Ney \\ Lehrstuhl für Informatik 6 \\ RWTH Aachen University \\ D-52056 Aachen, Germany \\ $\{$ vilar, leusch, ney\}acs.rwth-aachen. de \\ Rafael E. Banchs \\ D. of Signal Theory and Communications \\ Universitat Politècnica de Catalunya \\ 08034 Barcelona, Spain \\ rbanchs@gps.t.sc. upc.edu
}

\begin{abstract}
We introduce a novel evaluation scheme for the human evaluation of different machine translation systems. Our method is based on direct comparison of two sentences at a time by human judges. These binary judgments are then used to decide between all possible rankings of the systems. The advantages of this new method are the lower dependency on extensive evaluation guidelines, and a tighter focus on a typical evaluation task, namely the ranking of systems. Furthermore we argue that machine translation evaluations should be regarded as statistical processes, both for human and automatic evaluation. We show how confidence ranges for state-of-the-art evaluation measures such as WER and TER can be computed accurately and efficiently without having to resort to Monte Carlo estimates. We give an example of our new evaluation scheme, as well as a comparison with classical automatic and human evaluation on data from a recent international evaluation campaign.
\end{abstract}

\section{Introduction}

Evaluation of machine translation (MT) output is a difficult and still open problem. As in other natural language processing tasks, automatic measures which try to asses the quality of the translation can be computed. The most widely known are the Word Error Rate (WER), the Position independent word Error Rate (PER), the NIST score (Doddington, 2002) and, especially in recent years, the BLEU score (Papineni et al., 2002) and the Translation Er- ror Rate (TER) (Snover et al., 2005). All of theses measures compare the system output with one or more gold standard references and produce a numerical value (score or error rate) which measures the similarity between the machine translation and a human produced one. Once such reference translations are available, the evaluation can be carried out in a quick, efficient and reproducible manner.

However, automatic measures also have big disadvantages; (Callison-Burch et al., 2006) describes some of them. A major problem is that a given sentence in one language can have several correct translations in another language and thus, the measure of similarity with one or even a small amount of reference translations will never be flexible enough to truly reflect the wide range of correct possibilities of a translation. ${ }^{1}$ This holds in particular for long sentences and wide- or open-domain tasks like the ones dealt with in current MT projects and evaluations.

If the actual quality of a translation in terms of usefulness for human users is to be evaluated, human evaluation needs to be carried out. This is however a costly and very time-consuming process. In this work we present a novel approach to human evaluation that simplifies the task for human judges. Instead of having to assign numerical scores to each sentence to be evaluated, as is done in current evaluation procedures, human judges choose the best one out of two candidate translations. We show how this method can be used to rank an arbitrary number of systems and present a detailed analysis of the statistical significance of the method.

\footnotetext{
${ }^{1}$ Compare this with speech recognition, where apart from orthographic variance there is only one correct reference.
} 


\section{State-of-the-art}

The standard procedure for carrying out a human evaluation of machine translation output is based on the manual scoring of each sentence with two numerical values between 1 and 5 . The first one measures the fluency of the sentence, that is its readability and understandability. This is a monolingual feature which does not take the source sentence into account. The second one reflects the adequacy, that is whether the translated sentence is a correct translation of the original sentence in the sense that the meaning is transferred. Since humans will be the end users of the generated output, ${ }^{2}$ it can be expected that these human-produced measures will reflect the usability and appropriateness of MT output better than any automatic measure.

This kind of human evaluation has however additional problems. It is much more time consuming than the automatic evaluation, and because it is subjective, results are not reproducible, even from the same group of evaluators. Furthermore, there can be biases among the human judges. Large amounts of sentences must therefore be evaluated and procedures like evaluation normalization must be carried out before significant conclusions from the evaluation can be drawn. Another important drawback, which is also one of the causes of the aforementioned problems, is that it is very difficult to define the meaning of the numerical scores precisely. Even if human judges have explicit evaluation guidelines at hand, they still find it difficult to assign a numerical value which represents the quality of the translation for many sentences (Koehn and Monz, 2006).

In this paper we present an alternative to this evaluation scheme. Our method starts from the observation that normally the final objective of a human evaluation is to find a "ranking" of different systems, and the absolute score for each system is not relevant (and it can even not be comparable between different evaluations). We focus on a method that aims to simplify the task of the judges and allows to rank the systems according to their translation quality.

\section{Binary System Comparisons}

The main idea of our method relies in the fact that a human evaluator, when presented two different translations of the same sentence, can normally choose the best one out of them in a more or less

\footnotetext{
${ }^{2}$ With the exception of cross-language information retrieval and similar tasks.
}

definite way. In social sciences, a similar method has been proposed by (Thurstone, 1927).

\subsection{Comparison of Two Systems}

For the comparison of two MT systems, a set of translated sentence pairs is selected. Each of these pairs consists of the translations of a particular source sentence from the two systems. The human judge is then asked to select the "best" translation of these two, or to mark the translations to be equally good. We are aware that the definition of "best" here is fuzzy. In our experiments, we made a point of not giving the evaluators explicit guidelines on how to decide between both translations. As a consequence, the judges were not to make a distinction between fluency and adequacy of the translation. This has a two-fold purpose: on the one hand it simplifies the decision procedure for the judges, as in most of the cases the decision is quite natural and they do not need to think explicitly in terms of fluency and adequacy. On the other hand, one should keep in mind that the final goal of an MT system is its usefulness for a human user, which is why we do not want to impose artificial constraints on the evaluation procedure. If only certain quality aspects of the systems are relevant for the ranking, for example if we want to focus on the fluency of the translations, explicit guidelines can be given to the judges. If the evaluators are bilingual they can use the original sentences to judge whether the information was preserved in the translation.

After our experiment, the human judges provided feedback on the evaluation process. We learned that the evaluators normally selected the translation which preserved most of the information from the original sentence. Thus, we expect to have a slight preference for adequacy over fluency in this evaluation process. Note however that adequacy and fluency have shown a high correlation ${ }^{3}$ in previous experiments. This can be explained by noting that a low fluency renders the text incomprehensible and thus the adequacy score will also be low.

The difference in the amount of selected sentences of each system is an indicator of the difference in quality between the systems. Statistics can be carried out in order to decide whether this difference is statistically significant; we will describe this in more detail in Section 3.4.

\footnotetext{
${ }^{3}$ At least for "sensible" translation systems. Academic counter-examples could easily be constructed.
} 


\subsection{Evaluation of Multiple Systems}

We can generalize our method to find a ranking of several systems as follows: In this setting, we have a set of $n$ systems. Furthermore, we have defined an order relationship "is better than" between pairs of these systems. Our goal now is to find an ordering of the systems, such that each system is better than its predecessor. In other words, this is just a sorting problem - as widely known in computer science.

Several efficient sorting algorithms can be found in the literature. Generally, the efficiency of sorting algorithms is measured in terms of the number of comparisons carried out. State-of-the-art sorting algorithms have a worst-case running time of $\mathcal{O}(n \log n)$, where $n$ is the number of elements to sort. In our case, because such binary comparisons are very time consuming, we want to minimize the absolute number of comparisons needed. This minimization should be carried out in the strict sense, not just in an asymptotic manner.

(Knuth, 1973) discusses this issue in detail. It is relatively straightforward to show that, in the worst case, the minimum number of comparisons to be carried out to sort $n$ elements is at least $\lceil\log n !\rceil$ (for which $n \log n$ is an approximation). It is not always possible to reach this minimum, however, as was proven e.g. for the case $n=12$ in (Wells, 1971) and for $n=13$ in (Peczarski, 2002). (Ford Jr and Johnson, 1959) propose an algorithm called merge insertion which comes very close to the theoretical limit. This algorithm is sketched in Figure 1. There are also algorithms with a better asymptotic runtime (Bui and Thanh, 1985), but they only take effect for values of $n$ too large for our purposes (e.g., more than 100). Thus, using the algorithm from Figure 1 we can obtain the ordering of the systems with a (nearly) optimal number of comparisons.

\subsection{Further Considerations}

In Section 3.1 we described how to carry out the comparison between two systems when there is only one human judge carrying out this comparison. The comparison of systems is a very time consuming task. Therefore it is hardly possible for one judge to carry out the evaluation on a whole test corpus. Usually, subsets of these test corpora are selected for human evaluations instead. In order to obtain a better coverage of the test corpus, but also to try to alleviate the possible bias of a single evaluator, it is advantageous to have several evaluators carrying out the comparison between two systems. However, there are two points that must be considered.

The first one is the selection of sentences each human judge should evaluate. Assume that we have already decided the amount of sentences $m$ each evaluator has to work with (in our case $m=100$ ). One possibility is that all human judges evaluate the same set of sentences, which presumably will cancel possible biases of the evaluators. A second possibility is to give each judge a disjunct set of sentences. In this way we benefit from a higher coverage of the corpus, but do not have an explicit bias compensation.

In our experiments, we decided for a middle course: Each evaluator receives a randomly selected set of sentences. There are no restrictions on the selection process. This implicitly produces some overlap while at the same time allowing for a larger set of sentences to be evaluated. To maintain the same conditions for each comparison, we also decided that each human judge should evaluate the same set of sentences for each system pair.

The other point to consider is how the evaluation results of each of the human judges should be combined into a decision for the whole system. One possibility would be to take only a "majority vote" among the evaluators to decide which system is the best. By doing this, however, possible quantitative information on the quality difference of the systems is not taken into account. Consequently, the output is strongly influenced by statistical fluctuations of the data and/or of the selected set of sentences to evaluate. Thus, in order to combine the evaluations we just summed over all decisions to get a total count of sentences for each system.

\subsection{Statistical Significance}

The evaluation of MT systems by evaluating translations of test sentences - be it automatic evaluation or human evaluation - must always be regarded as a statistical process: Whereas the outcome, or score $R$, of an evaluation is considered to hold for "all" possible sentences from a given domain, a test corpus naturally consists of only a sample from all these sentences. Consequently, $R$ depends on that sample of test sentences. Furthermore, both a human evaluation score and an automatic evaluation score for a hypothesis sentence are by itself noisy: $\mathrm{Hu}-$ man evaluation is subjective, and as such is subject to "human noise", as described in Section 2. Each automatic score, on the other hand, depends heavily on the ambiguous selection of reference translations. Accordingly, evaluation scores underly a probability 
1. Make pairwise comparisons of $\lfloor n / 2\rfloor$ disjoint pairs of elements. (If $n$ is odd, leave one element out).

2. Sort the $\lfloor n / 2\rfloor$ larger elements found in step 1 , recursively by merge insertion.

3. Name the $\lfloor n / 2\rfloor$ elements found in step $2 a_{1}, a_{2}, \ldots, a_{\lfloor n / 2\rfloor}$ and the rest $b_{1}, b_{2}, \ldots, b_{\lceil n / 2\rceil}$, such that $a_{1} \leq a_{2} \leq \cdots \leq a_{\lfloor n / 2\rfloor}$ and $b_{i} \leq a_{i}$ for $1 \leq i \leq\lfloor n / 2\rfloor$. Call $b_{1}$ and the $a$ 's the "main chain".

4. Insert the remaining $b$ 's into the main chain, using binary insertion, in the following order (ignore the $b_{j}$ such that $\left.j>\lceil n / 2\rceil\right): b_{3}, b_{2} ; b_{5}, b_{4} ; b_{11}, \ldots, b_{6} ; \ldots ; b_{t_{k}}, \ldots, b_{t_{k-1}+1} ; \ldots$ with $t_{k}=\frac{2^{k+1}+(-1)^{k}}{3}$.

Figure 1: The merge insertion algorithm as presented in (Knuth, 1973).

distribution, and each evaluation result we achieve must be considered as a sample from that distribution. Consequently, both human and automatic evaluation results must undergo statistical analysis before conclusions can be drawn from them.

A typical application of MT evaluation - for example in the method described in this paper - is to decide whether a given MT system $X$, represented by a set of translated sentences, is significantly better than another system $Y$ with respect to a given evaluation measure. This outcome is traditionally called the alternative hypothesis. The opposite outcome, namely that the two systems are equal, is known as the null hypothesis. We say that certain values of $R_{X}, R_{Y}$ confirm the alternative hypothesis if the null hypothesis can be rejected with a given level of certainty, e.g. 95\%. In the case of comparing two MT systems, the null hypothesis would be "both systems are equal with regard to the evaluation measure; that is, both evaluation scores $R, R^{\prime}$ come from the same distribution $R_{0}$ ".

As $R$ is randomly distributed, it has an expectation $E[R]$ and a standard error $s e[R]$. Assuming a normal distribution for $R$, we can reject the null hypothesis with a confidence of $95 \%$ if the sampled score $R$ is more than 1.96 times the standard error away from the null hypothesis expectation:

$$
R \text { significant } \Leftrightarrow\left|E\left[R_{0}\right]-R\right|>1.96 \mathrm{se}\left[R_{0}\right]
$$

The question we have to solve is: How can we estimate $E\left[R_{0}\right]$ and $s e\left[R_{0}\right]$ ? The first step is that we consider $R$ and $R_{0}$ to share the same standard error $s e\left[R_{0}\right]=s e[R]$. This value can then be estimated from the test data. In a second step, we give an estimate for $E\left[R_{0}\right]$, either inherent in the evaluation measure (see below), or from the estimate for the comparison system $R^{\prime}$.

A universal estimation method is the bootstrap estimate: The core idea is to create replications of
$R$ by random sampling from the data set (Bisani and Ney, 2004). Bootstrapping is generally possible for all evaluation measures. With a high number of replicates, $s e[R]$ and $E\left[R_{0}\right]$ can be estimated with satisfactory precision.

For a certain class of evaluation measures, these parameters can be estimated more accurately and efficiently from the evaluation data without resorting to Monte Carlo estimates. This is the class of errors based on the arithmetic mean over a sentencewise score: In our binary comparison experiments, each judge was given hypothesis translations $e_{i, X}$, $e_{i, Y}$. She could then judge $e_{i, X}$ to be better than, equal to, or worse than $e_{i, Y}$. All these judgments were counted over the systems. We define a sentence score $r_{i, X, Y}$ for this evaluation method as follows:

$$
r_{i, X, Y}:=\left\{\begin{array}{ll}
+1 & e_{i, X} \text { is better than } e_{i, Y} \\
0 & e_{i, X} \text { is equal to } e_{i, Y} \\
-1 & e_{i, X} \text { is worse than } e_{i, Y}
\end{array} .\right.
$$

Then, the total evaluation score for a binary comparison of systems $X$ and $Y$ is

$$
R_{X, Y}:=\frac{1}{m} \sum_{i=1}^{m} r_{i, X, Y}
$$

with $m$ the number of evaluated sentences.

For this case, namely $R$ being an arithmetic mean, (Efron and Tibshirani, 1993) gives an explicit formula for the estimated standard error of the score $R_{X, Y}$. To simplify the notation, we will use $R$ instead of $R_{X, Y}$ from now on, and $r_{i}$ instead of $r_{i, X, Y}$.

$$
s e[R]=\frac{1}{m-1} \sqrt{\sum_{i=1}^{m}\left(r_{i}-R\right)^{2}} .
$$

With $x$ denoting the number of sentences where $r_{i}=1$, and $y$ denoting the number of sentences 
where $r_{i}=-1$,

$$
R=\frac{x-y}{m}
$$

and with basic algebra

$$
s e[R]=\frac{1}{m-1} \sqrt{x+y-\frac{(x-y)^{2}}{m}} .
$$

Moreover, we can explicitly give an estimate for $E\left[R_{0}\right]$ : The null hypothesis is that both systems are "equally good". Then, we should expect as many sentences where $X$ is better than $Y$ as vice versa, i.e. $x=y$. Thus, $E\left[R_{0}\right]=0$.

Using Equation 4, we calculate $s e[R]$ and thus a significance range for adequacy and fluency judgments. When comparing two systems $X$ and $Y$, we assume for the null hypothesis that $s e\left[R_{0}\right]=$ $s e\left[R_{X}\right]$ and $E\left[R_{0}\right]=E\left[R_{Y}\right]$ (or vice versa).

A very useful (and to our knowledge new) result for MT evaluation is that $s e[R]$ can also be explicitly estimated for weighted means - such as WER, PER, and TER. These measures are defined as follows: Let $d_{i}, i=1, \ldots, m$ denote the number of "errors" (edit operations) of the translation candidate $e_{i}$ with regard to a reference translation with length $l_{i}$. Then, the total error rate will be computed as

$$
R:=\frac{1}{L} \sum_{i=1}^{m} d_{i}
$$

where

$$
L:=\sum_{i=1}^{m} l_{i}
$$

As a result, each sentence $e_{i}$ affects the overall score with weight $l_{i}$ - the effect of leaving out a sentence with length 40 is four times higher than that of leaving out one with length 10 . Consequently, these weights must be considered when estimating the standard error of $R$ :

$$
s e[R]=\sqrt{\frac{1}{(m-1)(L-1)} \sum_{i=1}^{m}\left(\frac{d_{i}}{l_{i}}-R\right)^{2} \cdot l_{i}}
$$

With this Equation, Monte-Carlo-estimates are no longer necessary for examining the significance of WER, PER, TER, etc. Unfortunately, we do not expect such a short explicit formula to exist for the standard BLEU score. Still, a confidence range for BLEU can be estimated by bootstrapping (Och, 2003; Zhang and Vogel, 2004).

\begin{tabular}{|r|r|r|r|}
\hline & & Spanish & English \\
\hline \hline Train & Sentences & \multicolumn{2}{|c|}{$1.2 \mathrm{M}$} \\
\cline { 2 - 4 } & Words & $32 \mathrm{M}$ & $31 \mathrm{M}$ \\
\cline { 2 - 3 } & Vocabulary & $159 \mathrm{~K}$ & $111 \mathrm{~K}$ \\
\cline { 2 - 3 } & Singletons & $63 \mathrm{~K}$ & $46 \mathrm{~K}$ \\
\hline \hline Test & Sentences & 1117 & \multicolumn{1}{|c}{} \\
\cline { 2 - 3 } & Words & $26 \mathrm{~K}$ & \multicolumn{2}{|c}{72} \\
\cline { 2 - 3 } & OOV Words & \multicolumn{2}{|c}{} \\
\cline { 2 - 3 }
\end{tabular}

Table 1: Statistics of the EPPS Corpus.

\section{Evaluation Setup}

The evaluation procedure was carried out on the data generated in the second evaluation campaign of the TC-STAR project ${ }^{4}$. The goal of this project is to build a speech-to-speech translation system that can deal with real life data. Three translation directions are dealt with in the project: Spanish to English, English to Spanish and Chinese to English. For the system comparison we concentrated only in the English to Spanish direction.

The corpus for the Spanish-English language pair consists of the official version of the speeches held in the European Parliament Plenary Sessions (EPPS), as available on the web page of the European Parliament. A more detailed description of the EPPS data can be found in (Vilar et al., 2005). Table 1 shows the statistics of the corpus.

A total of 9 different MT systems participated in this condition in the evaluation campaign that took place in February 2006. We selected five representative systems for our study. Henceforth we shall refer to these systems as System A through System E. We restricted the number of systems in order to keep the evaluation effort manageable for a first experimental setup to test the feasibility of our method. The ranking of 5 systems can be carried out with as few as 7 comparisons, but the ranking of 9 systems requires 19 comparisons.

\section{Evaluation Results}

Seven human bilingual evaluators (6 native speakers and one near-native speaker of Spanish) carried out the evaluation. 100 sentences were randomly chosen and assigned to each of the evaluators for every system comparison, as discussed in Section 3.3. The results can be seen in Table 2 and Figure 2. Counts

\footnotetext{
${ }^{4}$ http: / / www.tc-star.org/
} 


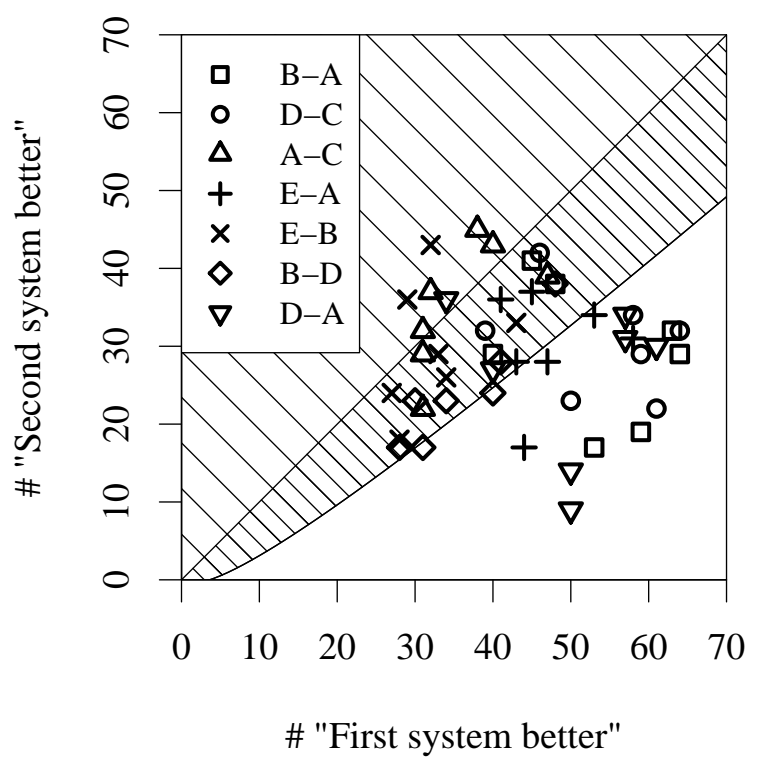

(a) Each judge.

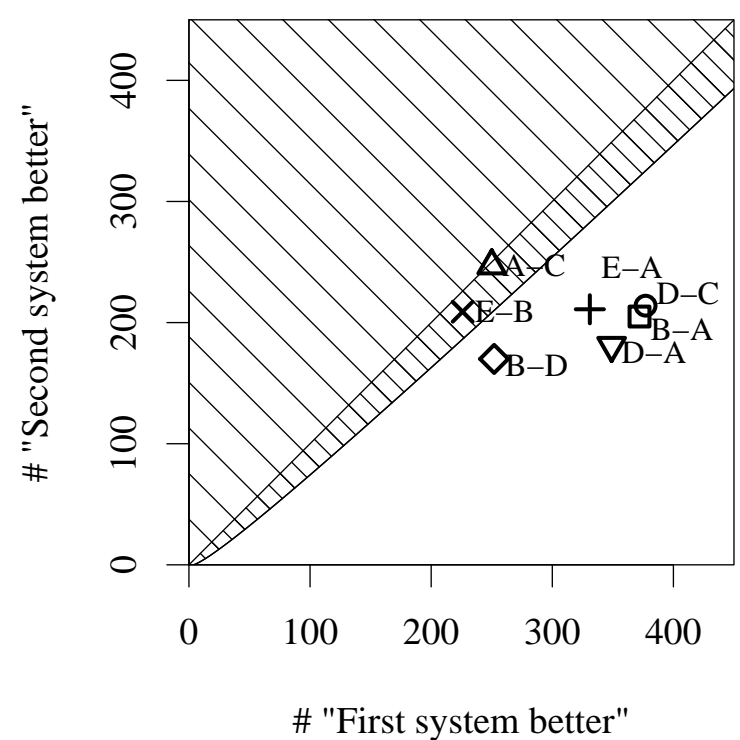

(b) All judges.

Figure 2: Results of the binary comparisons. Number of times the winning system was really judged "better" vs. number of times it was judged "worse". Results in hatched area can not reject null hypothesis, i.e. would be considered insignificant.

missing to 100 and 700 respectively denote "same quality" decisions.

As can be seen from the results, in most of the cases the judges clearly favor one of the systems. The most notable exception is found when comparing systems $\mathrm{A}$ and $\mathrm{C}$, where a difference of only 3 sentences is clearly not enough to decide between the two. Thus, the two bottom positions in the final ranking could be swapped.

Figure 2(a) shows the outcome for the binary comparisons separately for each judge, together with an analysis of the statistical significance of the results. As can be seen, the number of samples (100) would have been too low to show significant results in many experiments (data points in the hatched area). In some cases, the evaluator even judged better the system which was scored to be worse by the majority of the other evaluators (data points above the bisector). As Figure 2(b) shows, "the only thing better than data is more data": When we summarize $R$ over all judges, we see a significant difference (with a confidence of 95\%) at all comparisons but two (A vs. C, and E vs. B). It is interesting to note that exactly these two pairs do not show a significant difference when using a majority vote strategy.

Table 3 shows also the standard evaluation met- rics. Three BLEU scores are given in this table, the one computed on the whole corpus, the one computed on the set used for standard adequacy and fluency computations and the ones on the set we selected for this task ${ }^{5}$. It can be seen that the BLEU scores are consistent across all data subsets. In this case the ranking according to this automatic measure matches exactly the ranking found by our method. When comparing with the adequacy and fluency scores, however, the ranking of the systems changes considerably: B D E C A. However, the difference between the three top systems is quite small. This can be seen in Figure 3, which shows some automatic and human scores for the five systems in our experiments, along with the estimated $95 \%$ confidence range. The bigger difference is found when comparing the bottom systems, namely System A and System C. While our method produces nearly no difference the adequacy and fluency scores indicate System C as clearly superior to System A. It is worth noting that the both groups use quite different translation approaches (statistical vs. rule-based).

\footnotetext{
${ }^{5}$ Regretfully these two last sets were not the same. This is due to the fact that the "AF Test Set" was further used for evaluating Text-to-Speech systems, and thus a targeted subset of sentences was selected.
} 


\begin{tabular}{|c|ccccccc|c|}
\hline Sys & E1 & E2 & E3 & E4 & E5 & E6 & E7 & $\sum$ \\
\hline A & 29 & 19 & 38 & 17 & 32 & 29 & 41 & 205 \\
B & $\mathbf{4 0}$ & $\mathbf{5 9}$ & $\mathbf{4 8}$ & $\mathbf{5 3}$ & $\mathbf{6 3}$ & $\mathbf{6 4}$ & $\mathbf{4 5}$ & $\mathbf{3 7 2}$ \\
\hline C & 32 & 22 & 29 & 23 & 32 & 34 & 42 & 214 \\
D & $\mathbf{3 9}$ & $\mathbf{6 1}$ & $\mathbf{5 9}$ & $\mathbf{5 0}$ & $\mathbf{6 4}$ & $\mathbf{5 8}$ & $\mathbf{4 6}$ & $\mathbf{3 7 7}$ \\
\hline A & 32 & $\mathbf{3 1}$ & 31 & $\mathbf{3 1}$ & $\mathbf{4 7}$ & 38 & 40 & $\mathbf{2 5 0}$ \\
C & $\mathbf{3 7}$ & 29 & $\mathbf{3 2}$ & 22 & 39 & $\mathbf{4 5}$ & $\mathbf{4 3}$ & 247 \\
\hline A & 36 & 28 & 17 & 28 & 34 & 37 & 31 & 211 \\
E & $\mathbf{4 1}$ & $\mathbf{4 7}$ & $\mathbf{4 4}$ & $\mathbf{4 3}$ & $\mathbf{5 3}$ & $\mathbf{4 5}$ & $\mathbf{5 8}$ & $\mathbf{3 3 1}$ \\
\hline B & 26 & 29 & 18 & 24 & $\mathbf{4 3}$ & $\mathbf{3 6}$ & 33 & 209 \\
E & $\mathbf{3 4}$ & $\mathbf{3 3}$ & $\mathbf{2 8}$ & $\mathbf{2 7}$ & 32 & 29 & $\mathbf{4 3}$ & $\mathbf{2 2 6}$ \\
\hline B & $\mathbf{3 4}$ & $\mathbf{2 8}$ & $\mathbf{3 0}$ & $\mathbf{3 1}$ & $\mathbf{4 0}$ & $\mathbf{4 1}$ & $\mathbf{4 8}$ & $\mathbf{2 5 2}$ \\
D & 23 & 17 & 23 & 17 & 24 & 28 & 38 & 170 \\
\hline A & $\mathbf{3 6}$ & 14 & 27 & 9 & 31 & 30 & 34 & 181 \\
D & 34 & $\mathbf{5 0}$ & $\mathbf{4 0}$ & $\mathbf{5 0}$ & $\mathbf{5 7}$ & $\mathbf{6 1}$ & $\mathbf{5 7}$ & $\mathbf{3 4 9}$ \\
\hline
\end{tabular}

Final ranking (best-worst): E B D A C

Table 2: Result of the binary system comparison. Numbers of sentences for which each system was judged better by each evaluator (E1-E7).

\begin{tabular}{|c|c|ccc|c|}
\hline Subset: & Whole & \multicolumn{3}{|c|}{ A+F } & Binary \\
\hline Sys & BLEU & BLEU & A & F & BLEU \\
\hline A & 36.3 & 36.2 & 2.93 & 2.46 & 36.3 \\
B & 49.4 & 49.3 & 3.74 & 3.58 & 49.2 \\
C & 36.3 & 36.2 & 3.53 & 3.31 & 36.1 \\
D & 48.2 & 46.8 & 3.68 & 3.48 & 47.7 \\
E & 49.8 & 49.6 & 3.67 & 3.46 & 49.4 \\
\hline
\end{tabular}

Table 3: BLEU scores and Adequacy and Fluency scores for the different systems and subsets of the whole test set. BLEU values in \%, Adequacy (A) and Fluency $(\mathrm{F})$ from 1 (worst) to 5 (best).

\section{Discussion}

In this section we will review the main drawbacks of the human evaluation listed in Section 2 and analyze how our approach deals with them. The first one was the use of explicit numerical scores, which are difficult to define exactly. Our system was mainly designed for the elimination of this issue.

Our evaluation continues to be time consuming. Even more, the number of individual comparisons needed is in the order of $\log (n !)$, in contrast with the standard adequacy-fluency evaluation which needs $2 n$ individual evaluations (two evaluations per system, one for fluency, another one for adequacy). For $n$ in the range of 1 up to 20 (a realistic number of systems for current evaluation campaigns) these two quantities are comparable. And actually each of our

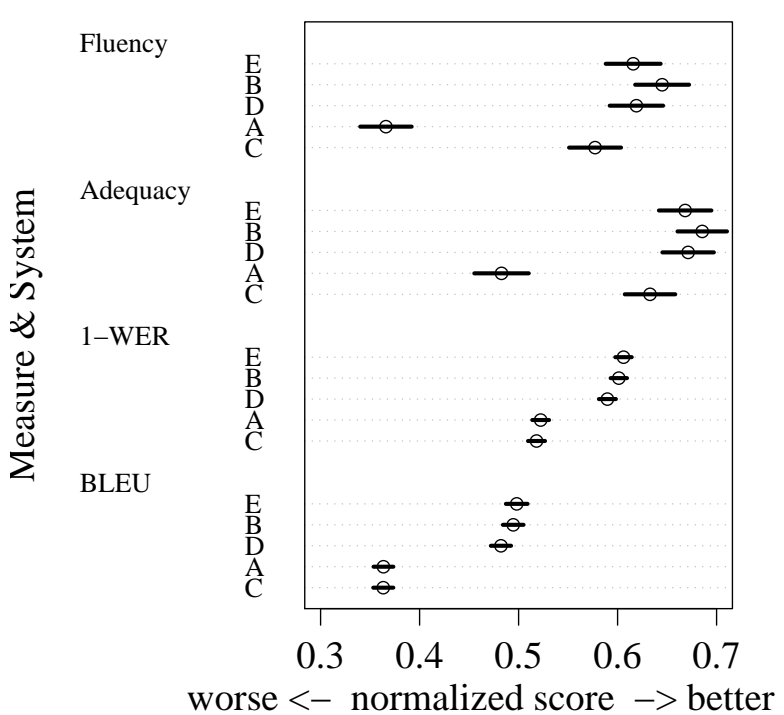

Figure 3: Normalized evaluation scores. Higher scores are better. Solid lines show the $95 \%$ confidence range. Automatic scores calculated on the whole test set, human scores on the $\mathrm{A}+\mathrm{F}$ subset.

evaluations should be simpler than the standard adequacy and fluency ones. Therefore the time needed for both evaluation procedures is probably similar.

Reproducibility of the evaluation is also an important concern. We computed the number of "errors" in the evaluation process, i.e. the number of sentences evaluated by two or more evaluators where the evaluators' judgement was different. Only in $10 \%$ of the cases the evaluation was contradictory, in the sense that one evaluator chose one sentence as better than the other, while the other evaluator chose the other one. In $30 \%$ of the cases, however, one evaluator estimated both sentences to be of the same quality while the other judged one sentence as superior to the other one. As comparison, for the fluencyadequacy judgement nearly one third of the common evaluations have a difference in score greater or equal than two (where the maximum would be four), and another third a score difference of one point ${ }^{6}$.

With respect to biases, we feel that it is almost impossible to eliminate them if humans are involved. If one of the judges prefers one kind of structure, there will a bias for a system producing such output, independently of the evaluation procedure. However, the suppression of explicit numerical scores eliminates an additional bias of evaluators. It has been observed that human judges often give scores within

\footnotetext{
${ }^{6}$ Note however that possible evaluator biases can have a great influence in these statistics.
} 
a certain range (e.g. in the mid-range or only extreme values), which constitute an additional difficulty when carrying out the evaluation (Leusch et al., 2005). Our method suppresses this kind of bias.

Another advantage of our method is the possibility of assessing improvements within one system. With one evaluation we can decide if some modifications actually improve performance. This evaluation even gives us a confidence interval to weight the significance of an improvement. Carrying out a full adequacy-fluency analysis would require a lot more effort, without giving more useful results.

\section{Conclusion}

We presented a novel human evaluation technique that simplifies the task of the evaluators. Our method relies on two basic observations. The first one is that in most evaluations the final goal is to find a ranking of different systems, the absolute scores are usually not so relevant. Especially when considering human evaluation, the scores are not even comparable between two evaluation campaigns. The second one is the fact that a human judge can normally choose the best one out of two translations, and this is a much easier process than the assessment of numerical scores whose definition is not at all clear. Taking this into consideration we suggested a method that aims at finding a ranking of different MT systems based on the comparison of pairs of translation candidates for a set of sentences to be evaluated.

A detailed analysis of the statistical significance of the method is presented and also applied to some wide-spread automatic measures. The evaluation methodology was applied for the ranking of 5 systems that participated in the second evaluation campaign of the TC-STAR project and comparison with standard evaluation measures was performed.

\section{Acknowledgements}

We would like to thank the human judges who participated in the evaluation. This work has been funded by the integrated project TC-STAR- Technology and Corpora for Speech-to-Speech Translation - (IST-2002-FP6-506738).

\section{References}

M. Bisani and H. Ney. 2004. Bootstrap estimates for confidence intervals in ASR performance evaluationx. IEEE ICASSP, pages 409-412, Montreal, Canada, May.
T. Bui and M. Thanh. 1985. Significant improvements to the Ford-Johnson algorithm for sorting. BIT Numerical Mathematics, 25(1):70-75.

C. Callison-Burch, M. Osborne, and P. Koehn. 2006. Reevaluating the role of BLEU in machine translation research. Proceeding of the 11th Conference of the European Chapter of the ACL: EACL 2006, pages 249256, Trento, Italy, Apr.

G. Doddington. 2002. Automatic evaluation of machine translation quality using n-gram co-occurrence statistics. Proc. ARPA Workshop on Human Language Technology.

B. Efron and R. J. Tibshirani. 1993. An Introduction to the Bootstrap. Chapman \& Hall, New York and London.

L. Ford Jr and S. Johnson. 1959. A Tournament Problem. The American Mathematical Monthly, 66(5):387-389.

D. E. Knuth. 1973. The Art of Computer Programming, volume 3. Addison-Wesley, 1st edition. Sorting and Searching.

P. Koehn and C. Monz. 2006. Manual and automatic evaluation of machine translation between european languages. Proceedings of the Workshop on Statistical Machine Translation, pages 102-121, New York City, Jun.

G. Leusch, N. Ueffing, D. Vilar, and H. Ney. 2005. Preprocessing and normalization for automatic evaluation of machine translation. 43rd ACL: Proc. Workshop on Intrinsic and Extrinsic Evaluation Measures for MT and/or Summarization, pages 17-24, Ann Arbor, Michigan, Jun.

F. J. Och. 2003. Minimum error rate training in statistical machine translation. Proc. of the 41st ACL, pages 160-167, Sapporo, Japan, Jul.

K. Papineni, S. Roukos, T. Ward, and W.-J. Zhu. 2002. Bleu: a method for automatic evaluation of machine translation. Proc. of the 40th ACL, pages 311-318, Philadelphia, PA, Jul.

M. Peczarski. 2002. Sorting 13 elements requires 34 comparisons. LNCS, 2461/2002:785-794, Sep.

M. Snover, B. J. Dorr, R. Schwartz, J. Makhoul, L. Micciulla, and R. Weischedel. 2005. A study of translation error rate with targeted human annotation. Technical Report LAMP-TR-126, CS-TR-4755, UMIACS-TR2005-58, University of Maryland, College Park, MD.

L. Thurstone. 1927. The method of paired comparisons for social values. Journal of Abnormal and Social Psychology, 21:384-400.

D. Vilar, E. Matusov, S. Hasan, R. Zens, and H. Ney. 2005. Statistical Machine Translation of European Parliamentary Speeches. Proceedings of MT Summit $X$, pages 259-266, Phuket, Thailand, Sep.

M. Wells. 1971. Elements of combinatorial computing. Pergamon Press.

Y. Zhang and S. Vogel. 2004. Measuring confidence intervals for the machine translation evaluation metrics. Proceedings of the 10th International Conference on Theoretical and Methodological Issues in Machine Translation, pages 4-6, Baltimore, MD. 